\title{
Relative fitness of carriers of the mitochondrial DNA mutation 3243A > G
}

\author{
Jukka S Moilanen and Kari Majamaa \\ Department of Neurology, University of Oulu, Oulu, Finland
}

\begin{abstract}
Deleterious point mutations in mitochondrial DNA (mtDNA) have been found in many human populations and always at a low frequency suggesting that they are under strong negative selection. It is assumed that this selection is caused by reduced genetic fitness of mutation carriers, but the fitness of carriers of any mtDNA mutation has not been determined. We estimated the reproductive disadvantage caused by the mitochondrial DNA mutation 3243A > G in a population-based group of female carriers $(n=32)$. The person-years method, Kaplan-Meier survival analysis and population statistics were used to estimate net reproduction rates of the mutation carriers and the general population. We found that women with 3243A > G reproduced at the same rate as women in the general population, suggesting that on average host-level selection against women harbouring the 3243A $>\mathrm{G}$ mutation is not strong. European Journal of Human Genetics (2001) 9, 59-62.
\end{abstract}

Keywords: mitochondrial encephalomyopathies; MELAS syndrome; population genetics; reproduction; net reproduction rate; selection

\section{Introduction}

Deleterious point mutations in mitochondrial DNA (mtDNA) have been found in many human populations and always at a low frequency suggesting that they are under strong negative selection. It has been suggested that this selection is caused by reduced genetic fitness of mutation carriers. ${ }^{1}$ One of these mutations is the heteroplasmic A-to-G transition at nucleotide 3243 in the tRN $A^{\text {Leu(UUR) }}$ gene $(3243 A>G)$, which is maternally inherited and causes the syndrome of mitochondrial encephalomyopathy with lactic acidosis and stroke-like episodes (MELAS). ${ }^{1}$ This syndrome is quite severe in phenotype and consequently it may be anticipated that the fertility of the mutation carriers should be reduced. We have previously screened for this mutation in a defined population, ${ }^{2}$ and here we compare the net reproduction rate of identified female carriers of the mutation with that in the general population to derive an estimate of host-level selection against the mutation.

Correspondence: Dr Kari Majamaa, University of Oulu, Department of Neurology, P.O. Box 5000, FIN-90014 University of Oulu, Finland. Tel: +3588315 4526; Fax: +35883154544; E-mail: kari.majamaa@oulu.fi Received 15 November 1999; revised 27 June 2000; accepted 11 July 2000

\section{Materials and methods}

Net reproduction rate (NRR) is a measure of the extent to which one female generation is reproducing itself. It can be interpreted as the average number of daughters a woman would have during her reproductive years, assuming fertility and mortality at given rates. $^{3}$ The relative reproductive capacity, or genetic fitness, of mutation carriers is given by the ratio of the net reproduction rate of mutation carriers $\left(N R R_{m}\right)$ over that of the general population $\left(N R R_{p}\right){ }^{4}$ We estimated retrospectively $\mathrm{NRR}_{\mathrm{m}}$ and $\mathrm{NRR}_{\mathrm{p}}$ in the population of the province of Oulu, Finland, (population 452367 in 1996) during the period 1January 1973 to 31 December 1996. Annual net reproduction rates of the general population were obtained from official population statistics. ${ }^{5-8}$

Net reproduction rate of $3243 \mathrm{~A}>\mathrm{G}$ carriers $\left(\mathrm{NRR}_{\mathrm{m}}\right)$ was estimated for women in 16 families which were not maternally related, based on the sequence variation in the $D$ loop of mtDNA. Eleven of these families had been found in a cross-sectional population-based screening for the mutation. ${ }^{2}$ The 92 women in the two generations defined by the proband and proband's mother were considered eligible for the analysis. Thirty-two women had been examined with respect to the mutation either because of symptoms of mitochondrial disease or voluntary participation following the identification of the mutation in other family members. 
Sixty women were not examined. The severity of disease was clinically estimated in each woman on a three-category scale, ${ }^{9}$ and that in unexamined women by using family history information as provided by the proband, and chart review whenever possible. All women harbouring the mutation were included in the estimation of $\mathrm{NRR}_{\mathrm{m}}$. We recorded the number of reproductive years defined as the age between 15 and 49 and coinciding with the years 1973-1996, and the number and date of live births during that period. From these data, age-specific birth rates at five-year intervals were estimated by using the person-years method. ${ }^{10} \mathrm{~A} 1: 1$ sex ratio in births was assumed, and therefore the number of daughters born per woman during each 5-year interval $\left(b_{x}\right.$ in Table 1) was calculated as $5 / 2=2.5$ times the total birth rate per person-year for the interval in question. The probability of survival to the midpoint of each 5-year interval $\left(I_{x}\right.$ in Table1) was estimated by Kaplan-Meier survival analysis of female mutation carriers in the 16 families (SAS statistical software6.12, LIFETEST procedure). $\mathrm{NRR}_{\mathrm{m}}$ was then calculated by multiplying, for each 5-year interval, the age-specific birth rate by the probability of survival to the midpoint of the interval and summing the products over all intervals (Table1). Since $I_{x}$ and $b_{x}$ are subject to sampling error and $N R R$ is a sum of $I_{x} b_{x}$ 's, the standard error (SE) of $N R R_{m}$ was calculated by using variance formulae for products and sums of independent random variables, ${ }^{11}$ and $95 \%$ confidence interval for $\mathrm{NRR}_{\mathrm{m}}$ was cal culated as $\mathrm{NRR}_{\mathrm{m}} \pm 1.96 \mathrm{SE}\left(\mathrm{NRR}_{\mathrm{m}}\right)$.

The ratio of $N R R_{m}$ and $N R R_{p}$ represents the relative fitness accurately only if the generation time is not markedly different between the mutation carriers and the general population. ${ }^{4}$ Therefore, the mean of age-specific fertility distribution $(m),{ }^{3}$ a commonly used measure of the timing of fertility, was calculated for both groups. For mutation carriers, $m$ was calculated by using the person-years method, ${ }^{10}$ and for the general population by using official population statistics. ${ }^{5-8}$ These two means were then com-

Table 1 Net reproduction rate of women with the $3243 \mathrm{~A}>\mathrm{G}$ mutation

\begin{tabular}{|c|c|c|c|c|}
\hline $\begin{array}{l}\text { Age } \\
\text { group }\end{array}$ & $\begin{array}{l}\text { Representative } \\
\text { age }(x)\end{array}$ & $\begin{array}{l}\text { Probability } \\
\text { of survival } \\
\text { to age } \\
x\left(I_{x}\right)\end{array}$ & $\begin{array}{l}\text { Age-specific } \\
\text { birth rate } \\
\left(b_{x}\right)\end{array}$ & $\begin{array}{l}\text { Number of } \\
\text { births to } \\
\text { survivors of } \\
\text { age } x\left(I_{x} b_{x}\right)\end{array}$ \\
\hline $\begin{array}{l}15-19 \\
20-24 \\
25-29 \\
30-34 \\
35-39 \\
40-44 \\
45-49\end{array}$ & $\begin{array}{l}17.5 \\
22.5 \\
27.5 \\
32.5 \\
37.5 \\
42.5 \\
47.5\end{array}$ & $\begin{array}{l}1.0 \\
1.0 \\
1.0 \\
0.967 \\
0.967 \\
0.967 \\
0.906\end{array}$ & $\begin{array}{l}0.066 \\
0.307 \\
0.250 \\
0.295 \\
0.119 \\
0 \\
0\end{array}$ & $\begin{array}{l}0.066 \\
0.307 \\
0.250 \\
0.285 \\
0.115 \\
0 \\
0\end{array}$ \\
\hline Total & & & & 1.02 \\
\hline
\end{tabular}

$x$, the midpoint of the respective age group; $I_{x}$, the Kaplan-Meier survival estimate to age $x ; b_{x}$, age-specific birth rate calculated by using the person-years method; $l_{x} b_{x}$, the product of $l_{x}$ and $b_{x}$. The net reproduction rate is obtained as the sum of $l_{x} b_{x}{ }^{\prime} s$. pared by one-way analysis of variance (SAS statistical software6.12, GLM procedure).

\section{Results}

The annual net reproduction rate of the general population varied between 0.87 and 1.06 during the years 1973-1996, the median $\left(\mathrm{NRR}_{\mathrm{p}}\right)$ being 1.01. Thirty-two women were shown to be carriers of 3243A > G and, except for one, their reproductive period coincided with the years 1973-1996, the total of reproductive years being 519. Six of the 31 women had the MELAS syndrome, 13 had diabetes mellitus or hearing impairment but no MELAS syndrome, and the remaining women had milder symptoms or were unaffected. The carrier status was unknown for 60 women, and in this group a total of 464 reproductive years in 31 women coincided with the period 1973-1996. Two of these 31 women had the MELAS syndrome, six had diabetes mellitus or hearing impairment but no MELAS syndrome, and the rest were mildly affected or unaffected. The net reproduction rate calculated for the 31 mutation carriers $\left(\mathrm{NRR}_{\mathrm{m}}\right)$ was similar to $\mathrm{NRR}_{\mathrm{p}}$ (Table1). The 95\% confidence interval for $\mathrm{NRR}_{\mathrm{m}}$ was 0.75-1.29. The mean of the age-specific fertility distribution $(\mathrm{m})$ was 28.4 years for the general population and 28.0 years for the mutation carriers ( $P=0.83$ for difference).

\section{Discussion}

It has been suggested that the apparent negative selection against deleterious point mutations of mtDNA is caused by reduced genetic fitness of mutation carriers. ${ }^{1}$ We found, however, that the average fertility of carriers of the $3243 \mathrm{~A}>\mathrm{G}$ mutation was not reduced. The confidence interval for the estimated $N_{R R}$ was wide, but severe reduction was nevertheless excluded. This suggests that women with $3243 \mathrm{~A}>\mathrm{G}$ are generally affected only mildly or late in life, and thus they have as many children as women in the general population.

Our sample of families with 3243A > G was populationbased, but it was not possible to determine the carrier status of all women in these families. Selection bias due to presenting symptoms of mtDNA disease was possible and consequently women who were examined may have had more severe phenotypes than those who were not. The family history method does not detect all affected relatives, ${ }^{12}$ and therefore, it is possible that their number was, in fact, higher than we observed. It is unlikely, however, that the unexamined women had been more severely affected than the examined women. Thus accounting for these factors would most likely increase the estimate of $\mathrm{NRR}_{\mathrm{m}}$. The number of person-years in unexamined women was lower than that in examined women because many unexamined women belonged to the sibship of the proband's mother and consequently their reproductive years did not coincide with the observational period. 
Since the late 1960s fertility and mortality have been low in the Finnish population in contrast to historical natural fertility populations, where both fertility and mortality were high. This implies that the fitness of mutation carriers in historical populations may have been different from that in the present population, especially if morbidity and mortality from the mutation are age-dependent and the average age at childbearing has changed. Interestingly, we found that the generation time was not different between the mutation carriers and the general population and it was also similar to that estimated from a historical population. ${ }^{13}$ It is also possible that morbidity and mortality from the mutation have changed. Diabetes mellitus, a common phenotype associated with the $3243 \mathrm{~A}>\mathrm{G}$ mutation, may have proved fatal in earlier times but is now treatable. On the other hand, no effective treatment is available for other severe phenotypes caused by $3243 \mathrm{~A}>\mathrm{G}$, and therefore marked differences between present and historical populations in the survival of mutation carriers are unlikely.

Relative fitness near unity suggests that host-level selection against the $3243 \mathrm{~A}>\mathrm{G}$ mutation is weak. If selection against the mutation at host level is assumed to be identical against all mutation carriers, the mutation survives on the average for $1 /\left(1-\left(N R R_{m} / N R R_{p}\right)\right)$ generations before it is eliminated from a maternal line. ${ }^{4}$ The lower end of the confidence interval for $\mathrm{NRR}_{\mathrm{m}}$ suggests a minimum survival time of 3243A > G to be four generations, whereas the point estimate of $\mathrm{NRR}_{\mathrm{m}}$ would correspond to an infinite survival and nonexistent selection against the mutation.

However, it is possible that the intensity of selection is not similar against all mutation carriers. The mtDNA is present in several copies in each mitochondrion, and there are between hundreds and thousands of mitochondria per cell. For disease-causing mutations it is common that only a portion of the mtDNA molecules harbours the mutation, leading to heteroplasmy. Experiments with transmitochondrial cells have shown that the proportion of $3243 \mathrm{~A}>\mathrm{G}$ mutant genomes must exceed approximately $90 \%$ before normal respiratory chain function is impaired, ${ }^{14}$ indicating that cells harbouring 3243A > G are faced with negative selection at the cellular level if the heteroplasmy is higher than this threshold. At the host level, factors that would lead to negative selection include female infertility, miscarriages, female mortality before or during reproductive years, and psychosocial constraints imposed by the disease. Each factor is probably directly related to the overall severity of the phenotype, and thus the degree of the mutant heteroplasmy in tissues may partly determine the reproductive disadvantage. Then, the average fitness of mutation carriers, as estimated in this study, would be related to the proportion of carriers with high mutant heteroplasmy. Furthermore, the fate of the mutation in each maternal line would not necessarily be determined primarily by selection itself but by events that change the degree of heteroplasmy from one generation to the next. The mitochondria are transferred randomly to daughter cells at cell divisions resulting in a drift in the degree of heteroplasmy in successive cell generations. In the female germline, this phenomenon is thought to be accelerated by a bottleneck, in which only a small, random sample of the mtDNA molecules in the parent cell passes through and contributes to the entire population of mtDNA molecules in daughter cells, thus increasing the variance of heteroplasmy in the successive generations of individuals. $^{15,16}$ These phenomena can expose the mutation to selection by increasing the degree of heteroplasmy, or remove the mutation by decreasing the heteroplasmy.

In conclusion, we found that the genetic fitness of $3243 \mathrm{~A}>\mathrm{G}$ carriers was not reduced, as indicated by comparison of net reproduction rates of the mutation carriers and the general population. The observed low frequency of this mutation in population could still be explained by a mild uniform selection or sel ection that depends on the degree of the mutant mtDNA heteroplasmy. On the other hand, our results are even compatible with the hypothesis that there is no host-level selection at all. This would imply that some other factors were responsible for the low population frequency of the mutation.

\section{Acknowledgements}

This work was supported in part by grants from the Medical Research Council of the Academy of Finland and from the Sigrid Juselius Foundation.

\section{References}

1 Zeviani M, Tiranti V, Piantadosi C: Mitochondrial disorders. Medicine 1998; 77: 59-72.

2 Majamaa K, Moilanen JS, Uimonen $\mathrm{S}$ et al: Epidemiology of A3243G, the mutation for mitochondrial encephalomyopathy, lactic acidosis, and strokelike episodes: prevalence of the mutation in an adult population. Am J Hum Genet 1998; 63: 447-454.

3 Newell C: Methods and Models in Demography. John Wiley \& Sons: Chichester, 1994.

4 Cavalli-Sforza LL, Bodmer WF: The Genetics of Human Populations. WH Freeman and Company: San Francisco, 1971.

5 Central Statistical Office of Finland: Vital Statistics 1973, 1974, 1975. Helsinki, 1976-1978.

6 Central Statistical Office of Finland: Population 1976, ... 1986. Helsinki, 1978-1987.

7 Central Statistical Office of Finland: Structure of Population and Vital Statistics 1987, 1988. Helsinki, 1987-1988.

8 Statistics Finland: Vital Statistics 1989, ... 1996. Helsinki, 1992-1997.

9 Karvonen SL, Haapasaari KM, Kallioinen M, Oikarinen A, Hassinen IE, Majamaa K: Increased prevalence of vitiligo, but no evidence of premature ageing, in the skin of patients with bp 3243 mutation in mitochondrial DNA in the mitochondrial encephalomyopathy, lactic acidosis and stroke-like episodes syndrome (MELAS). Br J Dermatol 1999; 140: 634-639.

10 Kahn HA, Sempos CT: Statistical Methods in Epidemiology. Oxford University Press: New York, 1989.

11 Armitage P, Berry G: Statistical Methods in Medical Research, 2nd edn. Blackwell Scientific Publications: Oxford, 1990.

12 Weissman MM, Merikangas KR, John K, Wickramaratne P, Prusoff BA, Kidd KK: Family-genetic studies of psychiatric disorders. Developing technologies. Arch Gen Psychiatry 1986; 43: 1104-1116. 
13 Tremblay M, Vézina $\mathrm{H}$ : New estimates of intergenerational time intervals for the calculation of age and origin of mutations. Am J Hum Genet 2000; 66: 651-658.

14 Bentlage HA, Attardi G: Relationship of genotype to phenotype in fibroblast-derived transmitochondrial cell lines carrying the 3243 mutation associated with the MELAS encephalomyopathy: shift towards mutant genotype and role of mtDNA copy number. Hum Mol Genet 1996; 5: 197-205.
15 Jenuth JP, Peterson AC, Fu K, Shoubridge EA: Random genetic drift in the female germline explains the rapid segregation of mammalian mitochondrial DNA. Nat Genet 1996; 14: 146-151.

16 Marchington DR, Macaulay V, Hartshorne GM, Barlow D, Poulton $\mathrm{J}$ : Evidence from human oocytes for a genetic bottleneck in an mtDNA disease. Am J Hum Genet 1998; 63: 769-775. 